\title{
Massive pulmonary embolism masked as an episode of acute agitation in a psychiatric patient: a case report
}

\author{
Gabriele Cioni, Giacomo Betti, Antonio Vergara \\ Emergency Department, SS Cosma and Damiano Pescia Hospital, USL Toscana Centro, Pescia (PT), Italy
}

\begin{abstract}
Management of the psychiatric diseases' reacutization is a frequent occurrence in emergency medicine. During this coronavirus disease 2019 pandemic, a further increase in access to the emergency room was reported, and the reasons are numerous. Although the essential prerogative of the emergency department is to provide immediate clinical assistance by rapidly setting an effective diagnostic and therapeutic path, there are multiple obstacles to providing adequate care for Emergency Department patients with mental illness. In this report, we describe the case of a 65 -year-old female patient with severe schizophrenia who was evaluated in the emergency room for acute agitation masking subtle, persistent dyspnea. The possibility of an underlying medical cause should not be underestimated or completely forgotten due to the difficult approach to the psychiatric patient.
\end{abstract}

\section{Introduction}

Management of the psychiatric diseases' reacutization is a frequent occurrence in emergency medicine. Recent data published before the coronavirus disease 2019 (COVID-19) pandemic showed an increasing trend in Emergency Department (ED) access for psychiatric patients in Italian hospitals, reaching $2 \%$ of total access in $2015^{1}$ and $2.7 \%$ in 2017 ; $^{2}$ these data are comparable with other international studies, ${ }^{3}$ emphasizing the relevance of the problem.

During this COVID-19 pandemic, a further increase in access to the emergency room was reported, and the reasons are numerous. Primarily due to the increasing difficulty of patients to continue their care or to get in touch with therapists. ${ }^{4}$ Moreover, the COVID-19 out-

Correspondence: Gabriele Cioni, Emergency Department, SS Cosma and Damiano Pescia Hospital, USL Toscana Centro, Pescia (PT), Italy.

E-mail: gabrielec.83@gmail.com

Key words: Massive pulmonary embolism; COVID-19.

Contributions: the authors contributed equally.

Conflict of interests: the authors declare no potential conflict of interests.

Received for publication: 30 October 2020

Revision received: 22 November 2020.

Accepted for publication: 24 November 2020.

This work is licensed under a Creative Commons Attribution NonCommercial 4.0 License (CC BY-NC 4.0).

${ }^{\circ}$ Copyright: the Author(s), 2021

Licensee PAGEPress, Italy

Italian Journal of Medicine 2021; 15:74-76

doi:10.4081/itjm.2020.1414 break leads to concomitant psychological problems such as stress, anxiety, depression, insomnia, and fear, aggravating mental health needs at the global level. ${ }^{5}$

Although the essential prerogative of the emergency department is to provide immediate clinical assistance by rapidly setting an effective diagnostic and therapeutic path, there are multiple obstacles to providing adequate care for ED patients with mental illness. ${ }^{6,7}$

Recent studies have highlighted that factors underlying these obstacles are multiple, depending on environmental features, availability of resources, personnel, and other elements strictly dependent on the patient's characteristics. ${ }^{7}$ In particular, a Survey conducted in the Australian emergency departments highlighted that both medical and nursing staff presented relevant gaps in the knowledge and specific management skills of psychiatric patients. ${ }^{8}$ It is known that an underlying medical condition can cause acute agitation in a psychiatric patient, but its identification is not always easy. ${ }^{9}$

For this reason, an incorrect or delayed diagnosis of clinical conditions other than psychiatric pathology, which afflict this category of patients, represents a dangerous possibility.

In this report, we describe the case of a 65 -year-old female patient with severe schizophrenia who was evaluated in the emergency room for acute agitation masking subtle, persistent dyspnea.

\section{Case Report}

The patient was affected by schizophrenia, and she was followed by the specialist psychiatry service from youth, reporting frequent reacutizations. In recent times a progressive decline of cognitive functions, associated with frequent psychomotor agitation episodes, was described, leading to repeated hospitalizations and treat- 
ment modifications. At the time of evaluation, the patient was taking only quetiapine.

The complained dyspnea has been described as persistent for some weeks; however, the patient's clinical history collection was difficult, and the summary for the difficulty of communication.

At the time of the visit, the patient appeared agitated and scarcely available for the interview with the medical staff.

She did not present tachypnea at rest; however, she was fatigued after speaking a few words. Not tachycardic, she denied chest pain.

Vital signs were normal, with the exception of a slight reduction in arterial oxygen saturation in ambient air (95\%). Clinically, she showed no signs of suspicion of deep vein thrombosis in the lower limbs, and there were no signs of heart failure, such as lower limb edema or jugular turgor. Arterial blood gases showed normal $\mathrm{pCO}_{2}$ values and only mild hypoxemia $\left(\mathrm{pO}_{2}\right.$ 74). However, we found a sinus rhythm on the electrocardiogram, with incomplete right bundle branch block, and normal heart rate. Chest X-ray was within regular limits.

However, from a more accurate medical questionnaire with her relatives, the patient appeared to have significantly reduced her mobility, with long periods of bed rest. Prolonged immobilization, obesity, ${ }^{10}$ and antipsychotic therapy ${ }^{11}$ are well-known risk factors for developing deep vein thrombosis and pulmonary embolism.

Bed-side venous doppler ultrasound of the lower limbs, performed in the emergency room, confirmed a deep vein thrombosis in the right femoral vein, extending to the popliteal; the thrombus presented mixed echogenicity, as for subacute thrombosis. Due to the presence of respiratory insufficiency and venous thrombosis in the lower limbs, we decided not to perform the D-dimer dosage, but a computed tomographic (CT) angiography of the pulmonary circulation.

Subsequent CT scan showed bilateral pulmonary embolism involving the main arterial branches, segmental and subsegmental branches (Figure 1). On the echocardiogram, we found dilation of the right sections in the absence of pericardial effusion; the patient was, however, hemodynamically stable.

The patient was immediately anticoagulated and hospitalized to continue treatment and investigations.

\section{Discussion}

Venous thromboembolism represents an acute pathology of worldwide interest, and above all, a critical pathology in emergency medicine. ${ }^{12}$

A useful tool in the suspicion of this disorder is blood D-dimer, in accordance with current international guidelines $;{ }^{13}$ however, in this report, we decided not to dose the D-dimer, considering the evidence of clinical findings, in particular, the presence of respiratory failure associated with the discovery of proximal deep vein thrombosis of the lower limb, and immediately perform $\mathrm{CT}$ angiography of the pulmonary circulation. The execution of a bedside ultrasound performed by the same emergency room physician allowed to reduce the diagnosis time and reinforced the suspicion of pulmonary embolism.

Despite the awareness that the clinical presentation of pulmonary embolism can be elusive, recent reports point out that diagnostic delays ${ }^{14}$ and underestimations are very common, and the reasons can be multiple. ${ }^{15}$

However, the differential diagnosis of pulmonary embolism has recently become complicated considering the typical clinical presentation of COVID-19 pneumonia. ${ }^{16-18}$

Furthermore, it is well known that the management of the psychiatric patient with psychomotor agitation in the emergency room is extremely complex. The differential diagnosis underlying episodes of acute agitation is broad and sometimes difficult to execute. In particular, the primary etiological causes that can trigger such a disorder can be purely medical conditions,${ }^{19}$ such as metabolic, neurological, infectious, and toxicological alterations, altering the precarious balance of the psychiatric patient. ${ }^{20}$ Delay in diagnosing such a condition can have severe, and sometimes fatal consequences.

The psychiatric patient's management during a severe acute respiratory syndrome-related coronavirus disease 2 epidemic could represent a new chapter in medicine, whose scientific evidence is still scarce. While many resources have been used to control and eradicate the spread of the virus, much still remains to be done to implement the management of psychiatric disorders in this period, especially considering the numerous psychological consequences of this contingency in outpatient settings. ${ }^{21}$

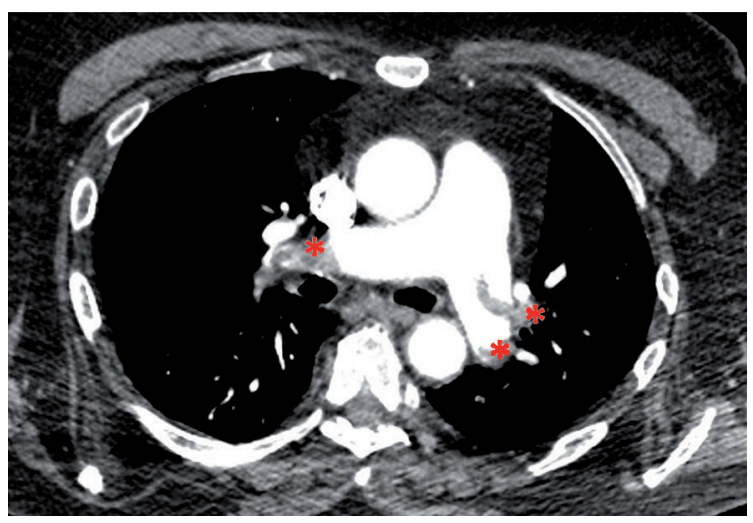

Figure 1. Pulmonary computed tomography revealing diffuse pulmonary embolism. Asterisks (*) reveal thrombosis of the main pulmonary arteries. 
In particular, the impact on psychiatric services systems has been described in different countries, including China, Italy, and the United States. ${ }^{4,22,23}$ The pandemic's consequences on the psychiatric patient can be directly related to having contracted the infection or indirectly due to the multiple psychological repercussions. Furthermore, the pandemic has been able to slow down psychiatric care, affect the subjects' family network, and the consequences can differ according to psychiatric patients' fragility. ${ }^{4}$ For example, patients suffering from substance abuse have been recognized as very vulnerable to the pandemic, both because many abuse substances have contributed to compromising the airways' functionality and because they are in poor social conditions, with little access to treatment. ${ }^{4}$ As for the elderly patient, many subjects were forced into isolation, with progressive loss of social relationships and exacerbations of anxiety disorders and depression. ${ }^{4}$

\section{Conclusions}

The management of the acute agitation episode of the psychiatric patient represents a critical moment in emergency medicine, especially in this pandemic phase. The possibility of an underlying medical cause should not be underestimated or completely forgotten due to the difficult approach to the psychiatric patient. While the COVID-19 pandemic management is the priority for health systems, many resources need to be deployed so that other acute clinical conditions are not underestimated or managed with delay.

\section{References}

1. Rapporto salute mentale. Analisi dei dati del sistema informativo per la salute mentale (SISM) 2015. Available from: http://www.salute.gov.it/imgs/C_17_pubblicazioni_ 2550_allegato.pdf

2. Rapporto salute mentale. Analisi dei dati del sistema informativo per la salute mentale (SISM) 2017. Available from: http://www.salute.gov.it/imgs/C_17_pubblicazioni_ 2841_allegato.pdf

3. Jelinek GA, Weiland TJ, Mackinlay C, et al. Knowledge and confidence of Australian emergency department clinicians in managing patients with mental health-related presentations: findings from a national qualitative study. Int J Emerg Med 2013;6:2.

4. Bojdani E, Rajagopalan A, Chen A, et al. COVID-19 pandemic: impact on psychiatric care in the United States. Psychiatry Res 2020;289:113069.

5. Dubey S, Biswas P, Ghosh R, et al. Psychosocial impact of COVID-19. Diabetes Metab Syndr 2020;14:779-88.

6. Dombagolla MHK, Kant JA, Lai FWY, et al. Barriers to providing optimal management of psychiatric patients in the emergency department (psychiatric patient management). Australas Emerg Care 2019;22:8-12.

7. Weiland TJ, Mackinlay C, Hill N, et al. Optimal management of mental health patients in Australian emergency departments: barriers and solutions. Emerg Med Australas 2011;23:677-88.

8. Sivakumar S, Weiland TJ, Gerdtz MF, et al. Mental health-related learning needs of clinicians working in Australian emergency departments: a national survey of self-reported confidence and knowledge. Emerg Med Australas 2012;23:697-711.

9. Chennapan K, Mullinax S, Anderson E, et al. Medical screening of mental health patients in the emergency department: a systematic review. J Emerg Med 2018;55:799-812.

10. Goldhaber SZ, Grodstein F, Stampfer MJ, et al. A prospective study of risk factors for pulmonary embolism in women. JAMA 1997;277:642.

11. Ogłodek EA, Just MJ, Grzesińska AD, et al. The impact of antipsychotics as a risk factor for thromboembolism. Pharmacol Rep 2018;70:533-9.

12. Di Nisio M, van Es N, Büller HR. Deep vein thrombosis and pulmonary embolism. Lancet 2016;388:3060-73.

13. Konstantinides SV, Meyer G, Becattini C, et al.; The Task Force for the diagnosis and management of acute pulmonary embolism of the European Society of Cardiology (ESC). 2019 ESC Guidelines for the diagnosis and management of acute pulmonary embolism developed in collaboration with the European Respiratory Society (ERS): The Task Force for the diagnosis and management of acute pulmonary embolism of the European Society of Cardiology (ESC). Eur Respir J 2019;54:1901647.

14. Elliott CG, Goldhaber SZ, Jensen RL. Delays in diagnosis of deep vein thrombosis and pulmonary embolism. Chest 2005; 128:3372-6.

15. Wilson E, Phair J, Carnevale M, Koleilat I. Common reasons for malpractice lawsuits involving pulmonary embolism and deep vein thrombosis. J Surg Res 2020;245:212-6.

16. Cioni G, Bellandi G, Bertolini S, et al. A multisystem approach by bed-side ultrasound in patients with COVID19 infection: a case series. Ital J Med 2020;14:106-11.

17. Cioni G. A pulmonary embolism-like pattern for the early identification of patients with SARS-CoV2 interstitial pneumonia: a case report. Ital J Emerg Med 2020;9:74-7.

18. Huang C, Wang Y, Li X, et al. Clinical features of patients infected with 2019 novel coronavirus in Wuhan, China. Lancet 2020;395:497.

19. Garriga M, Pacchiarotti I, Kasper S, et al. Assessment and management of agitation in psychiatry: Expert consensus. World J Biol Psychiatry 2016;17:86-128.

20. Gottlieb M, Long B, Koyfman A. Approach to the agitated emergency department patient. J Emerg Med 2018;54:447-57.

21. Torales J, O'Higgins M, Castaldelli-Maia JM, Ventriglio A. The outbreak of COVID-19 coronavirus and its impact on global mental health. Int $\mathrm{J}$ Soc Psychiatry 2020;66:317-20.

22. Cui LB, Wang XH, Wang HN. Challenges facing coronavirus disease 2019: Psychiatric services for patients with mental disorders. Psychiatry Clin Neurosci 2020 [Epub ahead of print].

23. Percudani M, Corradin M, Moreno M, et al. Mental health services in Lombardy during COVID-19 outbreak. Psychiatry Res 2020;288 [Epub ahead of print]. 